\title{
A Long-Duration Propulsive Lunar Landing Testbed
}

\author{
Krishna Shankar, Kevin Peterson, Heather Jones, Justin Moidel and William "Red" Whittaker
}

\begin{abstract}
Affordable test articles for descent and landing are crucial for developing commercial lunar landing capability. To ensure successful lunar landing, flight software must be tested over mission-length durations on hardware exhibiting dynamics analogous to those of true flight articles. Energetic and structural constraints typically preclude affordable longduration lander tests.

This paper details a first-in-kind propulsive lunar lander testbed designed for long-duration testing. The hardware and software on this platform exhibit dynamics that closely approximate those of real lunar landers and are exhibited to operate stably and indefinitely. The platform requires minimal infrastructure and is low cost to operate.

Platform configuration is outlined and state estimation for indefinite duration testing is detailed.
\end{abstract}

\section{INTRODUCTION}

Manned and robotic lunar missions of the 1960s set the technical stage for operations on the Moon, but high cadence, affordable access has not been realized. The last mission to soft-land on the Moon was Luna 24, in 1976. Since then, governments have sent scientific missions to orbit [1] and impact [2] the Moon, but none to land. Commerce has blossomed in Earth orbit, with satellites for weather, GPS and communications becoming a part of daily life, but no commercial entity has explored beyond Earth orbit.

Development of long-duration, dynamically faithful, lowcost testbeds is critical to commercial lunar enterprise. This paper details a first-in-kind propulsive lunar lander testbed, Black Magic, designed for long-duration hardware in the loop attitude control testing. Where other platforms compromise dynamics, duration, or price, Black Magic exhibits control and estimation for indefinite duration.

Lightweight aluminum honeycomb construction enables propulsion from shop-air without compromising dynamic response (Section II). The propulsion and inertia of the platform mimic the dynamics of the lander in development at Carnegie Mellon (Section I-B). Long-duration attitude estimation is accomplished using vision-based updates simulating star tracker output to stabilize inertial measurements (Section III). The resulting system is demonstrated to exhibit stable long-duration operation suitable for control experimentation (Section IV). The paper concludes in Section V.

\section{A. Related Work}

Three degree of freedom (DoF) simulators are commonly used to prototype and develop satellite attitude control. Air-

Krishna Shankar, kshanka2@illinois.edu, is with the Department of Aerospace Engineering at the University of Illinois at Urbana-Champaign. Kevin Peterson, Heather Jones, William "Red" Whittaker, \{kp, hjones, red\}@andrew.cmu.edu, and Justin Moidel, jmoidel@alumni.cmu.edu, are with Carnegie Mellon's Robotics Institute. bearing designs are often used to suspend test articles on a central pivot point [3], [4], [5], [6], [7]. This minimizes external torques and provides nearly friction-free motion. These platforms are often suspended on large zero-friction air bearings. These platforms are massive and preclude use of low-pressure propulsive sources.

Rocket thruster-based gravity off-loading enables 6 DoF flight tests [8]. Resisting Earth gravity requires significant fuel and therefore limits test duration. Operational, safety, and infrastructure requirements increase testing cost. Because of the short test duration, inertial-only sensing is employed and would drift unacceptably for long-duration tests.

Several 6 DoF platforms operate with minimal infrastructure. NASA-JPL's formation control testbed consists of an attitude platform supported by an air-bearing, placed on a wheeled base [9]. The platform uses on-board cold gas to generate thrust, so cannot be used for mission duration tests.

Fan based gravity off-loading is proposed to test navigation and control for $6 \mathrm{DoF}$ hopping robots [10]. In this configuration, fans provide thrust to offset Earth gravity while another form of thrust will be developed to provide platform control. While long-duration testing is enabled by the use of electric power, ducted fan dynamics differ significantly from those of propulsive thrusters appropriate to space.

No platform exists that exhibits the combination of accurate dynamics, long-duration operation, without significant infrastructure.

\section{B. Lunar Landing Spacecraft}

The lunar landing spacecraft in development by Carnegie Mellon University has a single, centrally located main engine and smaller, peripherally located thrusters that make up the attitude control system (ACS). The ACS thrusters are pulsed, with thrust modulated by varying the pulse width. The engines used for the ACS have been successfully operated in pulsed mode throughout a long duration flight using a minimum pulse width of $80 \mathrm{~ms}$. When the lander fuel tanks are empty, which is very close to the case during terminal descent and landing, the moments of inertia of the spacecraft are $I_{x x}=I_{y y}=449 \mathrm{kgm}^{2}$ and $I_{z z}=1002 \mathrm{kgm}^{2}$, and the thruster torque is $\tau_{x}=\tau_{y}=490 \mathrm{Nm}$ and $\tau_{z}=$ $200 \mathrm{Nm}$ (with the z-axis pointing up through the main thruster). Examining acceleration about a single axis and assuming initial rotational velcities of zero, the resulting angular acceleration is $63 \mathrm{deg} / \mathrm{s}^{2}$ about the $\mathrm{x}$-axis, $63 \mathrm{deg} / \mathrm{s}^{2}$ about the y-axis, or $11 \mathrm{deg} / \mathrm{s}^{2}$ about the $\mathrm{z}$-axis.

During transit to the Moon, pose will be roughly determined using Doppler measurements from Earth combined 
with star tracking and inertial measurement. As the lander approaches the Moon, star, inertial, visual, and radar measurements will dominate localization estimates. Final descent will be controlled by visually servoing to a selected landing site.

Black Magic replicates the sensing capability of the final descent incorporating camera and inertial measurements with a Kalman filter. Additional sensors for tracking the location of the Sun and Earth, measuring distance to the ground, and detecting obstacles will be added over time to develop the final sensor package for flight.

\section{BLACK MAGIC}

Black Magic, shown in Fig. 1 is a rotational motion platform designed to test spacecraft sensing and control. The platform is equipped with thrusters, sensors, and computer control and is currently restricted to orientation maneuvers. The platform is mass balanced and suspended on a bearing that accommodates $60^{\circ}$ of excursion in roll, pitch and yaw.

The platform operates in a controlled indoor environment where spotlights represent the Sun and Earth. To simulate star tracking, fiducial markers are placed around the platform and optically tracked. The tracker produces a quaternion attitude measurement with with rates and error characteristics similar to those of a star tracker. These measurements are filtered and sent to a motion controller. The platform executes attitude trajectories with high fidelity.

\section{A. Mechanical Configuration}

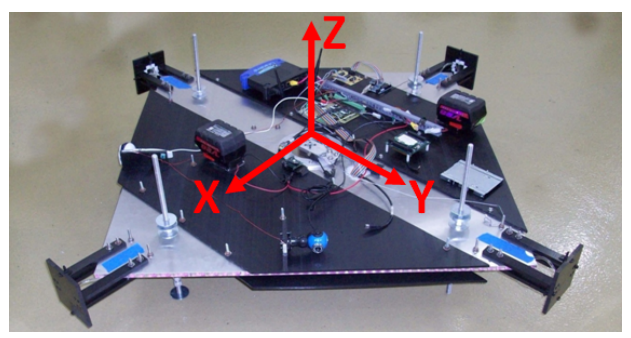

Fig. 1. Black Magic Platform

Black Magic's core is 40" x 40" x 3/8" aluminum honeycomb with .0205052 Permatreat aluminum face sheets. This core is exceptionally lightweight and high-strength. Electronics and sensors are mounted on Polyethylene sheet to enable straightforward transfer to other platforms.

The platform rests on a pin and cone bearing at the center of the honeycomb core. The pin and cone afford $60^{\circ}$ motion in roll and pitch and $360^{\circ}$ motion in yaw. Both pin and socket are hardened steel, resulting in good rotational freedom, low friction and durability (Fig. 2(b)).

Twelve cold gas thrusters actuate three degrees of rotational freedom. Four thrusters (two each, positive and negative) produce rotational motion around each axis. The thrusters are pulsed by $24 \mathrm{~V}$ solenoid valves at a minimum pulse width of $100 \mathrm{~ms}$ and are paired to produce pure moments. The system is partitioned into two clusters of four valves, and two clusters of two valves mounted at the four corners of the structure. Polyurethane tubing feeds compressed air to each thruster nozzle (Fig. 2(a)).

When two thrusters are firing, each produces $2.77 \mathrm{~N}$ of thrust. The thruster moment arm is $0.78 \mathrm{~m}$, giving a thruster torque of $2.16 \mathrm{Nm}$. The moments of inertia (in $\mathrm{kgm}^{2}$ ) of the platform are $I_{x x}=1.99, I_{y y}=1.90$ and $I_{z z}=3.82$ [11]. Assuming initial rotational velocities of zero, this thrust moment can produce angular accelerations of $62 \mathrm{deg} / \mathrm{s}^{2}$ about the $\mathrm{x}$-axis, $65 \mathrm{deg} / \mathrm{s}^{2}$ about the y-axis, or $32 \mathrm{deg} / \mathrm{s}^{2}$ about the z-axis. These accelerations match very well with the dynamics of the lander discussed in Section I-B for the $\mathrm{x}$ - and $\mathrm{y}$-axes. The correspondence for the acceleration about the z-axis is not as good, but for experiments in which the yaw rotation is of particular interest, the effective control authority about the z-axis of Black Magic can be reduced by decreasing the duty cycle for the yaw thrusters.

The platform is mass-balanced by adding weight to standoff poles located along each of the primary axes of the platform. This weight can be adjusted to simulate imbalance in mass distribution.

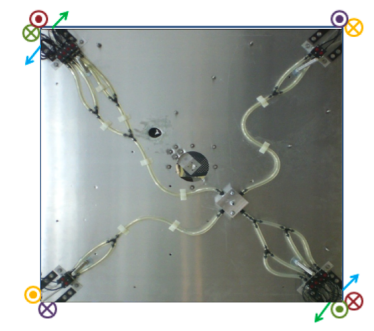

(a)

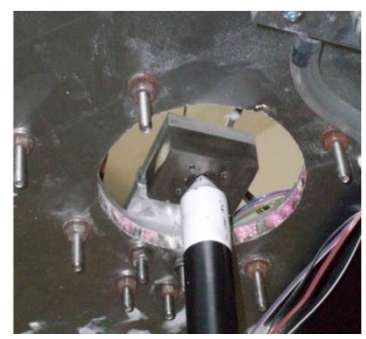

(b)
Fig. 2. (a) Compressed air is supplied to the Black Magic platform from a single air hose connection. Polyurethane tubing brings air out to individual thrusters. Symbols at the corners specify thrust direction, with color indicating coupled pairs. (b) A hardened steel pin sits in a hardened steel cup mounted to the center of Black Magic.

\section{B. Sensors}

Black Magic's sensors emulate those of a spacecraft. An Inertial Measurement Unit (IMU) provides high frequency measurements of attitude rates and accelerations. A downward looking camera tracks fiducial markers [12] to simulate star tracking, a direct measure of attitude. A Kalman Filter fuses measurements from these sensors to provide high-rate, stable estimates of pose for control (see Sec. III).

The platform utilizes a Crista IMU which samples MEMS gyroscopes and accelerometers mounted on orthogonal axes to provide $300 \mathrm{deg} / \mathrm{s}$ angular rate and $10 \mathrm{~g}$ acceleration data. The stationary IMU exhibits drift of less than $1 \mathrm{deg} / \mathrm{hour}$ after calibration.

Absolute attitude is provided by a downward facing camera. The camera tracks a pattern of reacTIVision fiducial markers below the platform and performs a non-linear least squares error minimization to recover attitude at $1 \mathrm{~Hz}$.

Two cameras provide measurements analogous to Earth and Sun tracking. Utilization of these measurements remains future work, although the sensor models for incorporating them are described below. 


\section{AtTitude Estimation}

Attitude estimation is accomplished by fusing high frequency rate measurements from gyroscopes with low frequency attitude measurements from the Earth, stars, and Sun $^{1}$ trackers[13]. A filtering scheme mixes measurements from these sensors to leverage their relative strengths and weaknesses. Latency due to limited computational capacity must be accounted for - this is particularly important for image processing.

\section{A. Sensor Models}

1) Inertial Measurements: IMUs consist of accelerometers and gyroscopes, and measure angular rates and accelerations about three perpendicular axes in the body frame of the platform. As the IMU rotates through space, the body frame measurements rotate out of the inertial reference frame (Fig. 3 ). To determine the inertial frame rotation of the platform, differential equations relating body-fixed angular rates to inertial frame measurements are solved in real time.

The quaternion representation of the attitude of the platform body frame with respect to the inertial frame is propagated by solving the following system of equations:

$$
\begin{aligned}
\dot{q}_{1} & =\frac{1}{2}\left(q_{0} \omega_{x}-q_{3} \omega_{y}+q_{2} \omega_{z}\right) \\
\dot{q_{2}} & =\frac{1}{2}\left(q_{3} \omega_{x}+q_{0} \omega_{y}-q_{1} \omega_{z}\right) \\
\dot{q_{3}} & =-\frac{1}{2}\left(q_{2} \omega_{x}-q_{1} \omega_{y}-q_{0} \omega_{z}\right) \\
\dot{q_{0}} & =-\frac{1}{2}\left(q_{1} \omega_{x}+q_{2} \omega_{y}+q_{3} \omega_{z}\right)
\end{aligned}
$$

where $q_{0}, q_{1}, q_{2}, q_{3}$ are the quaternion coordinates representing rotation and $\omega_{x}, \omega_{y}$, and $\omega_{z}$ are the body frame measurements.

Gyroscope error dynamics are highly correlated with motion - accelerations and moments applied to the gyroscpe will skew measurements and cause systematic bias:

$$
\begin{aligned}
\tilde{\omega}_{x} & =\left(1+S_{x}\right) \omega_{x}+M_{y} \omega_{y}+M_{z} \omega_{z}+B_{f} \\
& +B_{g x} a_{x}+B_{g z} a_{z}+B_{a x z} a_{y} a_{z}+n_{x}
\end{aligned}
$$

where $S_{x}$ is a scale factor error, $a_{x}, a_{y}, a_{z}$ are accelerations along the x-, y- and z-axes respectively. $M_{y}$ and $M_{z}$ are cross coupling coefficients, while $B_{f}$ is acceleration-independent bias. $B_{g x}, B_{g y}, B_{g z}$ are acceleration-dependent coefficients and $B_{a x z}, B_{a y z}, B_{a z z}$ are anisoelastic bias coefficients ${ }^{2}$ [13]. $n_{x}$ is a zero-mean random bias. When integrated, these biases cause the inertial frame estimate to drift.

Because the IMU is rarely located exactly at the center of rotation of the vehicle, rotation-induced accelerations generate bias even when thrust is not being applied. Thus, these motion-induced biases must be accounted for, regardless of the quality of the IMU hardware. For the purposes of our system, these biases are not considered individually, and are lumped into a single time varying drift-rate vector.

\footnotetext{
${ }^{1}$ The Sun is distinguished from other stars due to the significant difference in distance (and therefore difference in observation models).

${ }^{2}$ See [13] for equivalent models for $y$ - and $\mathrm{z}$-axis measurements
}

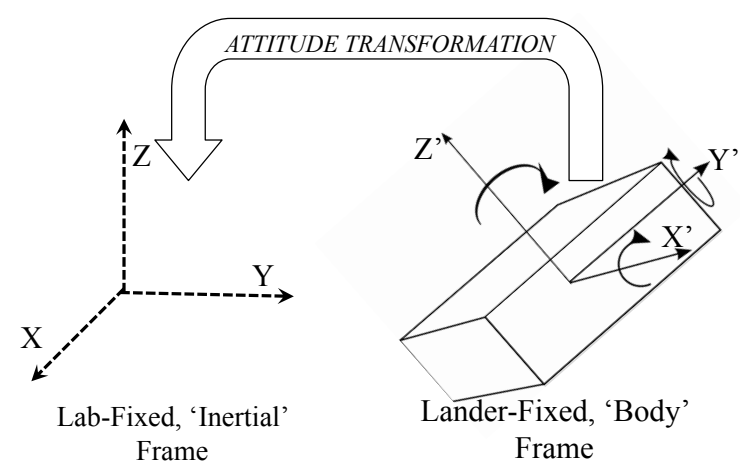

Fig. 3. Estimation reference frames. The IMU delivers measurements of 'Body' frame accelerations and rotation rates in the Lander local coordinates. The estimator determines attitude in the 'Inertial' reference frame. Body frame rates are rotated and then integrated to produce Inertial frame estimates.

2) Absolute Attitude Sensors: Many absolute attitude sensors in space work by measuring direction vectors to celestial bodies in sensor-fixed frames. Using knowledge of corresponding vectors in different reference frames (i.e. Earthfixed or Sun-fixed), attitude can be estimated. A minimum of two bodies must be tracked to yield measurements spanning three rotational degrees of freedom. For $n$ celestial objects being tracked, vector observations $\vec{\beta}_{i}$ and reference vectors $\overrightarrow{\gamma_{i}}$, there exists a rotation matrix ${ }^{3} L$ such that

$$
L \overrightarrow{\gamma_{i}}=\vec{\beta}_{i} \quad i=1,2, \ldots, n
$$

The observations are noisy. As a result a unique attitude matrix that satisfies the above condition cannot exist. Research ([15], [16]) has solved the 'Wahba' problem [17] of identifying an attitude estimate that achieves the least squared error.

\section{B. The Extended Kalman Filter}

Extended Kalman Filters (EKFs) enable best-fit (in the least square error sense) recursive estimation of system state. The evolution of state variables is modeled as a non-linear stochastic difference equation of the form

$$
x_{k+1}=f\left(x_{k}, u_{k}, w_{k}\right)
$$

where $x$ is the system state, $u$ is a control input, $w$ is white gaussian process noise, and $k$ is the time-step. An observation of the system is modeled as a non-linear mapping of the states:

$$
z_{k+1}=h\left(x_{k}, v_{k}\right)
$$

where $z$ is a measurement and $v$ is white, gaussian measurement noise.

The equations to be propagated with time to estimate the state are divided into a time-update cycle and a measurement update cycle. The time update is a prediction of state made

\footnotetext{
${ }^{3}$ This rotation matrix is effectively a linear transformation that takes any vector in the fixed-reference frame to the body frame - its columns are composed of the 'direction cosines', or cosines of the angles between rotated and fixed axes. see [14]
} 
only with knowledge of the system's previous state, control inputs and system dynamics. The measurement update is a correction made by comparing sensor measurements to the predicted state.

Time Update:

$$
\begin{aligned}
& x_{k+1}^{-}=f\left(x_{k}, u_{k}, 0\right) \\
& P_{k}^{-}=A_{k} P_{k} A_{k}^{T}+W_{k} Q_{k-1} W_{k}^{T}
\end{aligned}
$$

$$
\begin{aligned}
& \text { Mesurement Update: } \\
& K_{k}=P_{k-1} H^{T}\left(H P_{k-1} H^{T}+V R V^{T}\right)^{-1} \\
& x_{k}^{+}=x_{k-1}+K_{k}\left(y_{k}-h\left(x_{k-1}, 0\right)\right) \\
& P_{k}^{+}=\left(I-K_{k} H\right) P_{k-1}
\end{aligned}
$$

where $\mathrm{A}$ is the Jacobian of $f$ with respect to $x, W$ is the Jacobian of $f$ with respect to $w, H$ is the Jacobian of $h$ with respect to $x$ and $V$ is the Jacobian of $h$ with respect to $v . K$ is known as the 'Kalman Gain'-effectively a correction factor attained by comparing the state to the measurement, and $P$ is the error covariance matrix tracking the trustworthiness of the current estimate. Q is the process noise covariance matrix, and $\mathrm{R}$ is the measurement noise covariance matrix. More detailed descriptions of the EKF are available in [18] and [19].

\section{Numerical Integration of Rotation}

The model used on Black Magic derives from [20]. The state to be tracked consists of a quaternion and three drift rates:

$$
x=\left[\begin{array}{lllllll}
q_{1} & q_{2} & q_{3} & q_{0} & d_{1} & d_{2} & d_{3}
\end{array}\right]^{T}
$$

where $d_{1}, d_{2}$, and $d_{3}$ are the time-varying biases of the body frame measurements $\omega_{x}, \omega_{y}$, and $\omega_{z}$ respectively. Before every iteration of the filter, the state quaternion is normalized to unit length; maintaining unit norm in this way may seem crude, but is in fact optimal [21].

The high-frequency gyroscope rate data is used in the time-update step, to propagate the state. Solving the system of equations given in (1) is computationally expensive. If the direction of the spacecraft-fixed angular velocity vector is constant over each time-step, or if the quantity

$$
\overrightarrow{\triangle \theta}=\int_{t}^{t+\Delta t} \omega(\tau) d \tau
$$

is small, then

$$
q(t+\triangle t)=M(\overrightarrow{\triangle \theta}) q(t)
$$

where

$$
M(\overrightarrow{\triangle \theta})=\cos (|\overrightarrow{\triangle \theta}| / 2) I_{4 \times 4}+\frac{\sin (|\overrightarrow{\triangle \theta}| / 2)}{|\overrightarrow{\triangle \theta}|} \Omega(\overrightarrow{\triangle \theta})
$$

and

$$
\Omega(\vec{\omega})=\left[\begin{array}{cccc}
0 & \omega_{3} & -\omega_{2} & \omega_{1} \\
-\omega_{3} & 0 & \omega_{1} & \omega_{2} \\
\omega_{2} & -\omega_{1} & 0 & \omega_{3} \\
-\omega_{1} & \omega_{2} & -\omega_{3} & 0
\end{array}\right]
$$

The predictions of state made during the time-update stage involve applying (5) to the current state. Drift-rates follow a random walk and therefore do not change as a function of inertial data.

The Jacobian of the state transition function is given by

$$
\Phi\left(t, t_{0}\right)=\left[\begin{array}{cc}
\Theta\left(t, t_{0}\right) & \Psi\left(t, t_{0}\right) \\
0_{3 \times 4} & I_{3 \times 3}
\end{array}\right]
$$

where

$$
\begin{gathered}
\Theta\left(t, t_{0}\right)=M(\overrightarrow{\triangle \theta}) \Delta t \\
\Psi\left(t, t_{0}\right)=-\frac{1}{2}[M(\overrightarrow{\triangle \theta}) \Xi(\vec{q})] \triangle t \\
\Xi(\vec{q})=\left[\begin{array}{ccc}
q_{4} & -q_{3} & q_{2} \\
q_{3} & q_{4} & -q_{1} \\
-q_{2} & q_{1} & q_{4} \\
-q_{1} & -q_{2} & -q_{3}
\end{array}\right] \\
\Delta t=t-t_{0} \\
A_{k}=\Phi\left(t_{k}+\Delta t, t_{k}\right)
\end{gathered}
$$

\section{Measurement Equations}

Computations to perform direct attitude measurement (from e.g., star or fiducial trackers) are typically performed outside the attitude filter to produce a single, full-state representation of attitude. The resulting measurement function is the identity:

$$
h\left(x_{k}, 0\right)=\left[\begin{array}{llll}
q_{1} & q_{2} & q_{3} & q_{0}
\end{array}\right]^{T}
$$

Single-vector observations like Sun sensors measure a subspace of the full state-space. Supposing that the vector to a body in the reference frame of choice is $\left[\begin{array}{lll}\alpha & \beta & \gamma\end{array}\right]^{T}$, then the measurement function is:

$$
\begin{aligned}
& h\left(x_{k}, 0\right)= \\
& {\left[\begin{array}{c}
\alpha\left(2 q_{1}^{2}-\bar{q}+q_{0}^{2}\right)+\beta\left(2 q_{1} q_{2}+2 q_{3} q_{0}\right)+\gamma\left(2 q_{1} q_{3}-2 q_{2} q_{0}\right) \\
\alpha\left(2 q_{1} q_{2}-2 q_{3} q_{0}\right)+\beta\left(2 q_{2}^{2}-\bar{q}+q_{0}^{2}\right)+\gamma\left(2 q_{1} q_{0}+2 q_{2} q_{3}\right) \\
\left.\alpha\left(2 q_{1} q_{3}+2 q_{2} q_{0}\right)-\beta\left(2 q_{1} q_{0}-2 q_{2} q_{3}\right)+\gamma\left(2 q_{3}^{2}-\bar{q}+q_{0}^{2}\right)\right]
\end{array}\right]}
\end{aligned}
$$

where $\bar{q}=\sqrt{q_{1}^{2}+q_{2}^{2}+q_{3}^{2}}$. This function is the product of a rotation matrix written in terms of quaternion elements, and a reference direction vector.

\section{Estimation RESUlts}

\section{A. Turn-Table Tests}

To test the filter against data generated by the gyroscope and floor-tracker, data from these two sensors were collected during a series of simple rotations on a turn-table. The logged data was post-processed using software used to perform simulations. The test represented by the plots involved beginning at $0^{\circ}$ of roll, pitch and yaw, rotating to $-10^{\circ}$ in yaw, returning to $0^{\circ}$ yaw, rotating to $10^{\circ}$ yaw and finally coming to rest at $0^{\circ}$ yaw. See Figures 4 and 5 . 


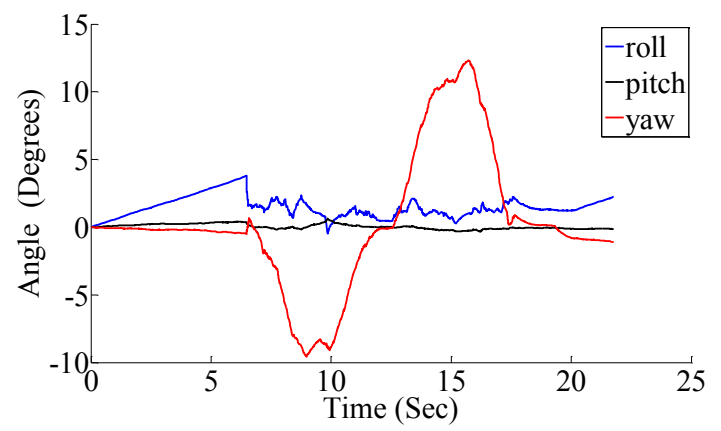

(a)

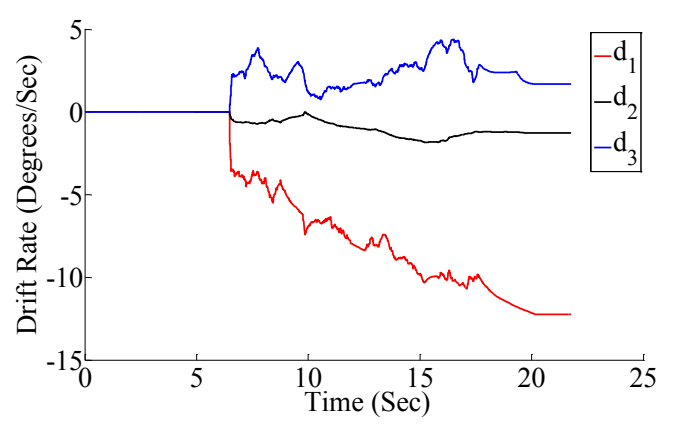

(b)

Fig. 4. Turn Table Tests. (a) Attitude (represented in Euler angles for readability). For the first five seconds, fiducial tracker estimates are unavailable and the attitude estimate drifts. Once absolute attitude measurements are included, drift rate estimates are determined and the estimate stabilizes. (b) corresponding drift rates for each axis.

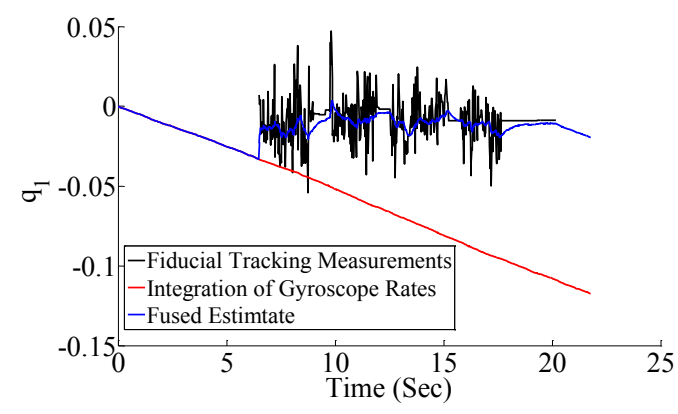

(a)

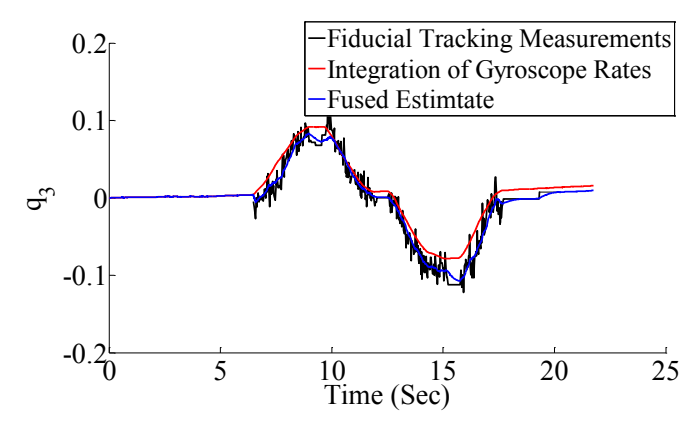

(c)

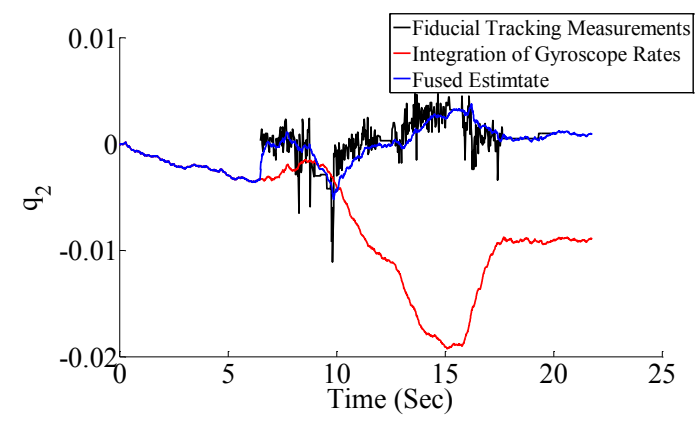

(b)

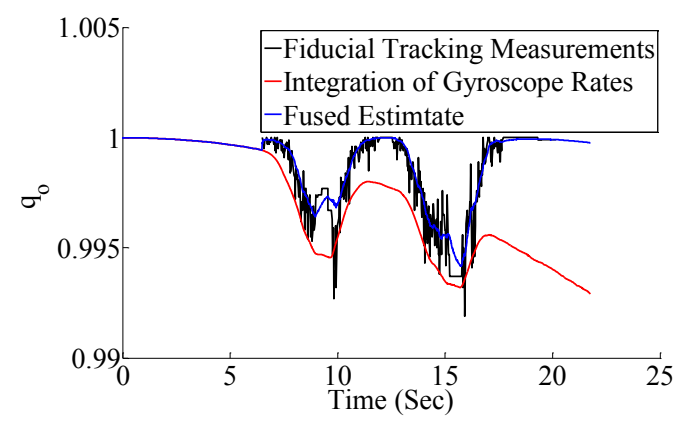

(d)

Fig. 5. Turn Table Tests. Attitude represented as a quaternion showing noisy camera data, smooth (but drifting) inertial measurements, and smooth, stable fused estimates.(a), (b) and (c) depict the elements of the vector portion of the quaternion, while (d) shows the varation in the scalar element, $q_{o}$

\section{B. Integrated Testing}

Experiments assessed the functionality of the filtering algorithm and the chosen sensors in real time on the Black Magic platform. The filter was tuned to ensure that smooth trajectory shape was taken from the integration of gyroscope rates, while the mean of the current attitude was determined by considering absolute attitude measurements.

To measure attitude accuracy, the platform was placed at an initial attitude and then displaced by an arbitrary amount and returned to the initial attitude. Error was measured to be better than 3 degrees in each axis independent of starting location 6.

Measurement latencies were handled by over expecting noise, and by keeping a time history of states. When a delayed measurement is available, it is compared to the state corresponding to the delay, and the resulting innovation is applied to the current state [22].

\section{CONCLUSiOn AND Future WORK}

This paper presented a test bed and algorithms for hardware-in-the-loop lunar landing tests. The platform exhibits thrust and inertia that are scaled about 1:250 to that of the lander being built by Carnegie Mellon University. The platform stably estimates attitude using sensors and algorithms analogous to those projected to guide spacecraft to the Moon. 


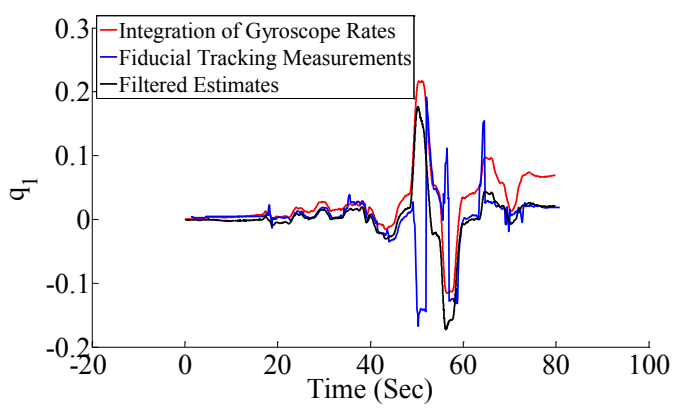

(a)

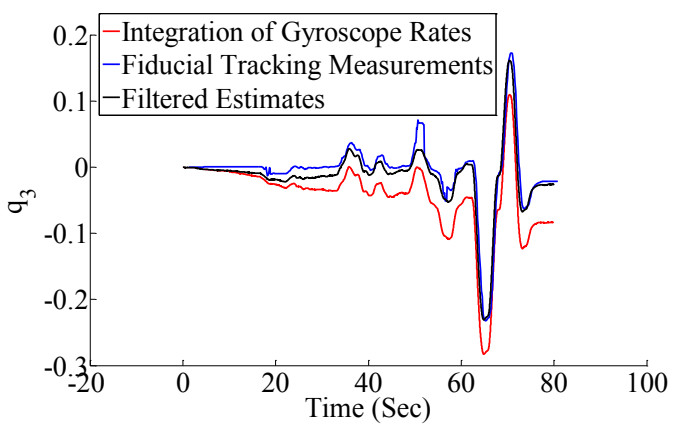

(c)

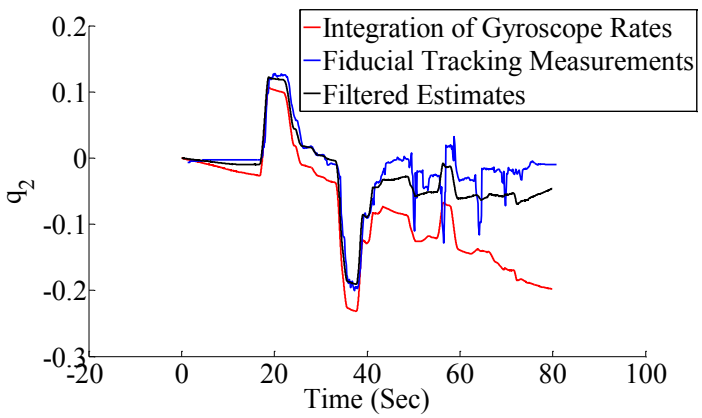

(b)

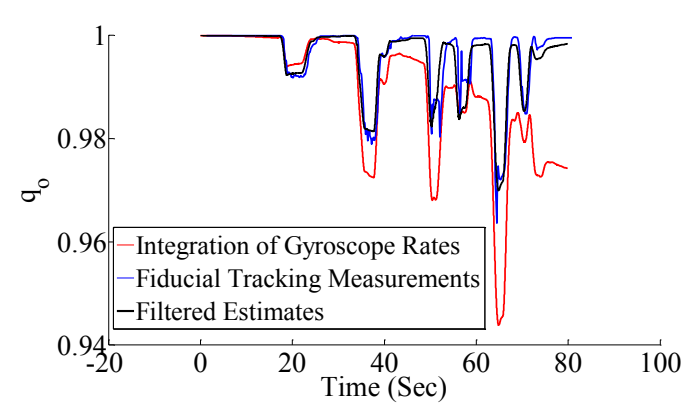

(d)

Fig. 6. Integrated test results showing estimation while Black Magic is in motion. Each plot shows individual elements of the attitude estimate over time.

In future tests, the Sun sensor, already implemented on the platform, will be filtered into the pose estimation. Black Magic will be suspended from a 3 DoF gantry crane to enable 6 DoF experimentation. The capability to sense position relative to features on the lunar surface will be added, and the planning of landing trajectories based on these features will be implemented. The control system will be tuned to optimize various parameters, such as fuel use, overshoot or settling time, and the control performance will be tested and analyzed.

\section{REFERENCES}

[1] C. R. Tooley et al., "Lunar Reconnaissance Orbiter Mission and Spacecraft Design,” Space Science Reviews, vol. 150, no. 1, pp. 23-62, 2010.

[2] A. Colaprete et al., "Water and More: An Overview of LCROSS Impact Results," in Lunar and Planetary Institute Science Conference Abstracts, vol. 41, March 2010, p. 979.

[3] J. L. Schwartz, M. A. Peck, and C. D. Hall, "Historical review of air-bearing spacecraft simulators," Journal of Guidance, Control, and Dynamics, vol. 26, no. 4, 2003.

[4] B. Kim et al., "Designing a low-cost spacecraft simulator," IEEE Control Systems Magazine, pp. 26-37, August 2003.

[5] The Distributed Spacecraft Attitude Control System Simulator: Development, Progress, Plans. Greenbelt, Maryland: NASA Goddard Space Flight Center, October 2003.

[6] N. H. Hansen, "Development of a computer balanced motion table: a ground testing facility for microsatellite attitude control systems," Master's thesis, University of Toronto, October 2000.

[7] B. Kim et al., "Designing a low-cost spacecraft simulator," Control Systems Magazine, IEEE, vol. 23, no. 4, pp. 26-37, 2003.

[8] B. Cohen et al., "Robotic lunar landers for science and exploration," in 41 st Lunar and Planetary Science Conference, The Woodlands, Texas, March 2010.
[9] D. P. Scharf et al., "Flight-like ground demonstration of precision formation flying spacecraft," in Techniques and Instrumentation for Detection of Exoplanets III, vol. 6693, September 2007.

[10] C. Dillow, "MIT-designed space hopper leaps across planetary surfaces," http://web.mit.edu/newsoffice/2010/talaris-0818.html, August 2010.

[11] J. Moidel, "Rotational motion platform for emulating spacecraft attitude control,' Master's thesis, Robotics Institute, Carnegie Mellon University, Pittsburgh, PA, August 2010.

[12] S. Jordà et al., "The reacTable," in Proceedings of the International Computer Music Conference, 2005, pp. 579-582.

[13] D. Titterton and J. L. Weston, Strapdown Inertial Navigation Technology, 2nd ed. Stevenage, United Kingdom: Institution of Electrical Engineers, 2004.

[14] L. Sciavicco and B. Siciliano, Modeling and Control of Robot Manipulators. McGraw-Hill Company, Inc., 2001.

[15] M. Shuster, "Maximum likelihood estimation of spacecraft attitude," Journal of the Astronautical Sciences, vol. 37, no. 1, pp. 79-88, January 1989.

[16] F. Markley, "Attitude determination using vector observations and the singular value decomposition," Journal of the Astronautical Sciences, vol. 36, no. 3, pp. 245-258, 1988.

[17] G. Wahba, "Problem 65-1: A least squares estimate of spacecraft attitude," SIAM Review, vol. 7, no. 3, p. 409, 1965.

[18] G. Welch and G. Bishop, "An introduction to the Kalman filter," University of North Carolina at Chapel Hill, Tech. Rep. TR 95-041, 2006.

[19] A. Gelb, Ed., Applied Optimal Estimation. The MIT press, 1974.

[20] E. Lefferts, F. Markley, and M. Shuster, "Kalman filtering for spacecraft attitude estimation," Journal of Guidance, Control, and Dynamics, vol. 5, no. 5, pp. 417-429, 1982.

[21] I. Bar-Itzhack, "Optimum normalization of a computed quaternion of rotation," IEEE Transactions on Aerospace and Electronic Systems, no. 2, pp. 401-402, 2007.

[22] R. van der Merwe, E. Wan, and S. Julier, "Sigma-point kalman filters for nonlinear estimation and sensor-fusion: Applications to integrated navigation," in Proceedings of the AIAA Guidance, Navigation \& Control Conference, 2004. 